\title{
ALGORITMO PARA PLANEAR TRAYECTORIAS DE ROBOTS MÓVILES, EMPLEANDO CAMPOS POTENCIALES Y ENJAMBRES DE PARTÍCULAS ACTIVAS BROWNIANAS
}

\author{
A PATH PLANNING ALGORITHM FOR MOBILE ROBOTS USING POTENTIAL FIELDS AND \\ SWARMS OF ACTIVE BROWNIAN PARTICLES \\ Helbert Eduardo Espitia Cuchango \\ Ing. Electrónico, Mecatrónico, Esp, Mag., Profesor Asistente \\ Universidad Distrital Francisco José de Caldas, Bogotá, Colombia \\ heespitiac@udistrital.edu.co \\ Jorge Iván Sofrony Esmeral \\ Ing. Eléctrico, MSc, PhD., Profesor Asistente \\ Universidad Nacional de Colombia, Bogotá, Colombia \\ jsofronye@unal.edu.co
}

Fecha de recepción: 3 de mayo de 2012

Fecha de aprobación: 2 de diciembre 2012

\section{RESUMEN}

En este documento se presenta la propuesta de un algoritmo para planear trayectorias, empleando un modelo de partículas activas brownianas. Existen varios métodos para planear trayectorias en robótica móvil, y uno de los más populares es el basado en campos potenciales artificiales; sin embargo, este método tiene la desventaja de presentar mínimos locales lo cual puede hacer que el robot no logre llegar al punto destino. Aunque ya se han realizado aplicaciones de enjambres de partículas para evadir mínimos locales, en la propuesta aquí presentada, se busca emplear un modelo compacto que permita planear la trayectoria, evadiendo mínimos locales.

Palabras clave: robótica móvil, planeación de trayectorias, partículas activas brownianas.

\begin{abstract}
This paper offers a proposal of an algorithm for path planning based on a model of active Brownian particles. There are several methods for trajectory planning in mobile robotics, and the most known is based on artificial potential fields; however this method has a disadvantage of local minima which can make the robot fails to reach the destination. Although applications have been made to avoid local minima using swarms of particles, this proposal seeks to use a compact model that allows the trajectory planning eluding local minima.
\end{abstract}

Keywords: mobile robotics, path planning, active Brownian particles. 


\section{INTRODUCCIÓN}

El comportamiento de muchos seres vivos depende de la interacción que se presenta con sus vecinos, lo cual es útil para buscar alimento y también, para evadir depredadores. El modelado de estos comportamientos ha sido objeto de atención en áreas como matemática, física, biología e ingeniería; adicionalmente, se ha buscado emplear este comportamiento para solucionar problemas en ingeniería.

La descripción del comportamiento de muchos entes biológicos, se caracteriza por exhibir movimiento cooperativo coordinado, tal como poblaciones de bacterias que presentan movimientos basados en quimiotaxis. También se observa este tipo de comportamiento en bandadas, cardúmenes, e incluso, en organismos como el zooplancton [1, 2, 3]. De los modelos más empleados para representar este comportamiento se tiene el de partículas autopropulsadas $[4,5]$, y de partícula activa browniana [6]. El segundo modelo considera un componente estocástico, a diferencia del primer tipo de modelo. Estos modelos tienen la característica de poder describir el movimiento de partículas con vorticidad (movimientos circulares alrededor de un punto). Por lo general, estos modelos suelen emplear potenciales de Morse para representar la interacción entre individuos; sin embargo, Erdmann Udo et al [7] presenta un modelo compacto que utiliza un potencial parabólico para describir este tipo de interacción.

En particular, los modelos de enjambres han sido empleados con exito para generar trayectorias de uno o varios robots, tal como se puede apreciar en Abdel Wahid M. H. M. [8].

La aplicación que se presenta en este documento, emplea modelos de partículas activas brownianas, donde se busca tener un algoritmo con pocos parámetros que incorpore una fuerza de auto-propulsión y un componente de movimiento browniano. Con este modelo, se espera explotar la característica de vorticidad que presentan las partículas. El algoritmo propuesto consiste en un movimiento traslacional de las partículas cuando estas no están un mínimo local y de un comportamiento de vorticidad (circular), cuando las partículas se encuentren en un mínimo local. El principio del algoritmo consiste en incrementar la fuerza de autopropulsión cuando el enjambre se encuentre en un mínimo local, de tal forma que las partículas puedan escapar de él y finamente, llegar al punto destino.

\section{PLANEACIÓN DE TRAYECTORIAS PARA ROBOTS MÓVILES}

Convencionalmente el problema de generación de trayectorias para un robot móvil indica que dado un robot y un ambiente, se puede determinar una trayectoria entre dos puntos específicos si el robot está libre de colisiones y si satisface cierto criterio de desempeño [9, 10].

Una primera clasificación de los métodos para planear de movimientos de robots móviles, es dinámica o estática [11]. En el caso dinámico, no se tiene información completa del espacio 
de trabajo y los obstáculos se consideran móviles. Para el caso estático, se tiene conocimiento completo del espacio de trabajo y los obstáculos no se mueven. En [12, 13, 14, 15], se puede apreciar algunas aplicaciones para generar trayectorias de robots móviles ante la presencia de obstáculos estáticos y dinámicos.

De los métodos para planear trayectorias, se tiene en un primer lugar, los basados en grafos entre los cuales se distinguen: grafos de visibilidad [16], diagramas de Voronoi [17], modelado del espacio libre [18], y descomposición en celdas [19, 20].

Otro tipo de métodos empleados para la planeación de trayectorias emplea un enfoque estocástico que da lugar al algoritmo de planeación aleatoria de trayectorias (Randomized Path Planner RPP) [21], al algoritmo de mapas probabilísticos (Probabilistic Road Map Method PRM) [22, 23], y al algoritmo de árboles de exploración rápida (Rapidly Exploring Random Tree RRT), [24].

Enfoques adicionales para planear trayectorias utilizan métodos heurísticos de optimización como algoritmos genéticos [25, 26, 27, 28], colonias de hormigas [29], quimiotaxis bacteriana [30], y optimización mediante enjambres de partículas [31].

\subsection{PLANEACIÓN DE TRAYECTORIAS, EMPLEANDO EL MÉTODO DE CAMPOS POTENCIALES}

El método de campos potenciales es una de las técnicas más populares en la generación de trayectorias para robots móviles, por ser una forma práctica de solucionar este problema. Entre los primeros desarrollos, existe el presentado por Khatib O. [32], que propone que los obstáculos y el robot tengan una carga eléctrica del mismo signo, con la finalidad de generar una repulsión, mientras que la meta tiene asociada una carga eléctrica de signo opuesto, para atraer el robot al punto destino. La propuesta presentada por Khatib O. [32], se denominó FIRAS (sigla en francés que se traduce al inglés como The Force Involving and Artificial Repulsion from the Surface Function).

En modo convencional, los potenciales atractivos y repulsivos se generan de forma separada y el potencial total del espacio de trabajo se obtiene como la suma de aquellos. Otra propuesta con este mismo enfoque, fue desarrollada por Krogh B. A. [33] y es Ilamada GPF/Generalized Potential Field). Para generar la trayectoria con estos potenciales, sólo se requiere calcular los gradientes; sin embargo, los campos potenciales pueden ser no convexos, presentando mínimos locales que dejaría estancado al robot en un punto diferente al objetivo. Otra desventaja es la estimación de los parámetros del campo potencial para lograr una adecuada evasión de obstáculos. Modificaciones al planteamiento original de campos potenciales se realizan, considerando las anteriores limitaciones; Volpe y Khosla [34], proponen las funciones potenciales super-cuadráticas (Super-quadric Artificial Potential Functions), donde se emplea un amplio conjunto de formas desde rectángulos hasta elipses que están descritos por fórmulas super-cuadráticas. Una propuesta basada en la solución de la ecuación de Laplace para transferencia de calor, es desarrollada 
de forma separada por Connolly [37], y adicionalmente por Kim y Khosla [35]; este método se implementa mediante funciones potenciales armónicas (Harmonic Potential Functions). En el caso de tener obstáculos en movimiento, S.S. Ge y Y.J. Cui [36], proponen un nuevo conjunto de funciones potenciales que dependen de la posición y de la velocidad de los obstáculos.

Un problema adicional identificado tanto por S.S. Ge y Y.J. Cui [36], y por R. Volpe y P. Khosla [34], aparece cuando el campo potencial de un obstáculo encierra el objetivo, lo cual ocurre cuando estos dos se encuentran muy cercanos. Este problema se denomina GNRON /Goals Nonreachable With Obstacle Nearby); los anteriores autores proponen en sus trabajos diferentes tipos de campos potenciales para solucionar este problema.

Los métodos presentados anteriormente, se denominaron campos potenciales locales, debido a su característica de adición de campos asociados a los obstáculos. En contraste con esta orientación, existen los campos potenciales globales donde se considera la totalidad de los obstáculos, como también el objetivo para construir el campo potencial como un todo y no como la adición de partes; con esta orientación, se busca eliminar el problema de los mínimos locales. Bajo este nuevo enfoque aparecen las funciones de navegación (Navigation Function), propuestas por Rimon y Koditschek [38], que son explícitamente diseñadas para presentar un único mínimo local en el objetivo, y si existe otro tipo de puntos de equilibrio, se enmascara (rellena). Para construir las funciones de navegación, es necesario conocer completamente el espacio de trabajo, con la desventaja de perder la simplicidad que presentan los otros métodos antes expuestos [39].

\subsection{MÉTODOS PARA EVADIR MÍNIMOS LOCALES EN CAMPOS POTENCIALES}

Un algoritmo desarrollado para evadir mínimos locales en campos potenciales, se presenta en Prahlad Vadakkepat et al [40], que se llama fuerza de escape EF (Escape Force), el cual consiste en determinar los mínimos locales para aplicar una fuerza adicional que le permita al robot, evitar el mínimo local.

Un método adicional para evadir mínimos locales, consiste en generar un objetivo virtual; en este caso, cuando el robot se encuentra en un mínimo, se adiciona un objetivo virtual en lugar del global, hasta que el robot logre salir de este punto [41]. Este enfoque se hace para que el robot pueda rodear obstáculos. Otro trabajo bajo una orientación similar, consiste en adicionar un obstáculo virtual [42].

Por último, Abdel Wahid M. H. M. et al [8] emplea un conjunto de agentes con comportamiento de enjambre y en particular con movimientos circulares, con la finalidad de evadir mínimos locales. Esta técnica se denomina Local Minimal Avoidance (LMA), o Local Minimal Escape (LME).

HELBERT EDUARDO ESPITIA CUCHANGO, JORGE IVÁN SOFRONY ESMERAL 


\section{MODELO EMPLEADO}

El modelo seleccionado para implementar el algoritmo de planeación de trayectorias, se encuentra basado en la forma de locomoción del zooplancton Daphnia que emplea movimientos circulares para buscar alimento. Con este modelo, se busca aprovechar la forma de locomoción con características de vorticidad ya que tal como se presenta en Abdel Wahid M. H. M. et al [8] esta puede ser una buena estrategia para evadir mínimos locales. El modelo seleccionado es:

$$
\begin{array}{r}
\frac{d \vec{r}_{i}}{d t}=\vec{v}_{i} \\
m_{i} \frac{d \vec{v}_{i}}{d t}=\vec{F}_{p r o, i}+\vec{F}_{i n t, i}+\vec{F}_{e s p, i}+\vec{F}_{e s t, i}
\end{array}
$$

Donde la primera ecuación corresponde al cálculo de posición $\vec{r}_{i}$ de la partícula, conociendo su velocidad $\vec{v}_{i}$. La segunda ecuación corresponde al cálculo de la velocidad de la partícula donde $m_{i}$ es la masa de la $i$-ésima partícula.

La fuerza de auto-propulsión se considera como:

$$
\vec{F}_{p r o, i}=\left(-\alpha+\beta\left|v_{i}\right|^{2}\right) \vec{v}_{i}
$$

El término anterior es muy similar al presente en la ecuación de Rayleigh que describe un comportamiento de ciclo límite. Cuando actúa sólo esta fuerza, la velocidad de las partículas en estado estable tiende a ser $\left|\vec{v}_{i}\right|=\sqrt{\alpha / \beta}[5,4]$. La fuerza de interacción de las partículas está dada por:

$$
\begin{array}{r}
\vec{F}_{i n t, i}=-a\left(\vec{r}_{i}-\vec{R}\right) \\
\vec{F}_{i n t, i}=-\frac{a}{N} \sum_{j=1}^{N}\left(\vec{r}_{i}-\vec{r}_{j}\right)
\end{array}
$$

Donde $\vec{R}$ corresponde al centro de masa del enjambre:

$$
\vec{R}=\frac{1}{N} \sum_{j=1}^{N} \vec{r}_{j}
$$

La información de los obstáculos y la meta se encuentra contenida en el campo potencial $U_{\text {esp }}$ donde se puede emplear cualquier tipo de funciones potenciales para su implementación; sin embargo, en esta propuesta se emplean potenciales parabólicos $U_{\text {obj }}$ para la meta y barrera de potencial exponencial para los obstáculos $U_{o b s ;}$; las expresiones de estos potenciales son:

$$
U_{o b j}(\vec{r})=k_{o b j} \operatorname{dis}\left(\vec{r}, \vec{r}_{o b j}\right)^{2}
$$




$$
U_{o b s}(\vec{r})=k_{o b s} \operatorname{dis}\left(\vec{r}, \vec{r}_{o b s}\right)^{-1}
$$

Donde $\vec{r}_{o b j}$ es la posición de la meta y $\vec{r}_{o b s}$ la posición del obstáculo. La función de distancia en coordenadas rectangulares es:

$$
\operatorname{dis}\left(\vec{r}, \vec{r}_{a}\right)=\sqrt{\left(x-x_{a}\right)^{2}+\left(y-y_{a}\right)^{2}}
$$

Considerando $M$ obstáculos, el potencial total se puede calcular como:

$$
U_{e s p}=U_{o b j}(\vec{r})+\sum_{m=1}^{M} U_{o b s, m}(\vec{r})
$$

La fuerza sobre cada partícula que se produce por el potencial $U_{e s p}$ es:

$$
\vec{F}_{e s p, i}=-k_{f} \nabla_{i} U_{e s p}\left(\vec{r}_{i}\right)
$$

Donde $k_{f}$ es una constante que pondera la influencia que tienen los obstáculos y la meta.

Al final se tiene la fuerza estocástica $\vec{F}_{\text {est }, i}=\vec{\xi}_{i}(t)$ que según Mikhailov Alexander S. et al [43] evita el colapso de los individuos, de tal forma que se puede omitir la interacción de repulsión de corto rango. Adicionalmente, Erdmann Udo et al [7] y Mikhailov Alexander S. et al [43], muestran que el término $\vec{\xi}(t)$ permite tener una transición entre un movimiento traslacional a rotacional, cuando se tienen las mismas condiciones iniciales en la posición y la velocidad para las partículas. Esta fuerza se describe mediante una distribución normal de media cero y varianza $D$.

\section{ANÁLISIS DEL MODELO}

En esta sección, se presenta un análisis aproximado para la evolución de la energía del enjambre y cómo se afecta el momento angular cuando la formación está cerca de un punto de equilibrio, es decir, cuando la posición media del enjambre $(\vec{R})$, se encuentra en un punto donde las fuerzas dadas por el campo potencial son cercanas a cero. En el caso mencionado anteriormente, se aprecia que las partículas presentan la capacidad de girar alrededor del punto de equilibrio (mínimo local), lo cual hace que el momento angular permanezca invariable. 


\subsection{ANÁLISIS DE ENERGÍA}

Siendo $K_{i}, U_{i}$ la energía cinética y potencial de la $i$-ésima partícula, las cuales están definidas como:

$$
K_{i}=\frac{1}{2} m_{i} v_{i}^{2}, \quad U_{i}=U_{i n t}\left(\vec{r}_{i}\right)+k_{f} U_{e s p}\left(\vec{r}_{i}\right)
$$

La energía de la $i$-ésima partícula $E_{i}=K_{i}+U_{i}$ y la energía total del enjambre corresponden a la suma de las energías de todas las partículas, donde $\alpha, \beta, a$ y $k_{f}$ son parámetros no negativos. Tomando la derivada para la energía de una partícula $E_{i}=K_{i}+U_{i}$ :

$$
\begin{array}{r}
\dot{E}_{i}=\frac{d}{d t}\left(\frac{1}{2} m_{i} v_{i}^{2}+U_{i n t}\left(\vec{r}_{i}\right)+k_{f} U_{e s p}\left(\vec{r}_{i}\right)\right) \\
\dot{E}_{i}=\left(\alpha-\beta\left\|\vec{v}_{i}\right\|^{2}\right)\left\|\vec{v}_{i}\right\|^{2}-k\left(\vec{r}_{i}-\vec{R}\right) \dot{\vec{R}}
\end{array}
$$

Sumando las contribuciones para cada partícula y considerando que $\sum_{i=1}^{N}\left(\vec{r}_{i}-\vec{R}\right)=0$, se tiene:

$$
\dot{E}=\sum_{i=1}^{N} \dot{E}_{i}=\sum_{i=1}^{N}\left(\alpha-\beta\left\|\vec{v}_{i}\right\|^{2}\right)\left\|\vec{v}_{i}\right\|^{2}
$$

En 13 si $\left\|\vec{v}_{i}\right\|=0$ o $\left\|\vec{v}_{i}\right\|^{2}=\alpha / \beta$ entonces la energía tiende a ser constante.

\subsection{ANÁLISIS DEL MOMENTO ANGULAR}

El objetivo de esta sección es mostrar que una vez que el enjambre ha llegado a un mínimo local, las partículas describen movimientos circulares alrededor de $\vec{R}$, el cual se encuentra en un mínimo. Con $\alpha, \beta, a$ y $k_{f}$ parámetros no negativos, el momento angular de la $i$-ésima partícula está dado por:

cuyo cambio en el tiempo es:

$$
\vec{L}_{i}=\vec{r}_{i} \times m_{i} \vec{v}_{i}
$$

$$
\begin{aligned}
\dot{\vec{L}}_{i} & =\vec{v}_{i} \times m_{i} \vec{v}_{i}+\vec{r}_{i} \times m_{i} \dot{\vec{v}}_{i} \\
& =\vec{r}_{i} \times m_{i} \dot{\vec{v}}_{i} \\
& =\vec{r}_{i} \times\left[\left(\alpha-\beta\left\|\vec{v}_{i}\right\|^{2}\right) \vec{v}_{i}-a\left(\vec{r}_{i}-\vec{R}\right)-k_{f} \vec{\nabla} U_{e s p}\left(\vec{r}_{i}\right)\right]
\end{aligned}
$$


Adicionando la variación del momento angular de todas las partículas del enjambre alrededor de su centro de masa, se tiene:

$$
\begin{aligned}
\dot{\vec{L}} & =\sum_{i=1}^{N} \dot{\vec{L}}_{i} \\
& =\sum_{i=1}^{N} \vec{r}_{i} \times\left[\left(\alpha-\beta\left\|\vec{v}_{i}\right\|^{2}\right) \vec{v}_{i}-a\left(\vec{r}_{i}-\vec{R}\right)-k_{f} \vec{\nabla} U_{e s p}\left(\vec{r}_{i}\right)\right]
\end{aligned}
$$

Dado que $\vec{r}_{i} \times \vec{r}_{j}+\vec{r}_{j} \times \vec{r}_{i}=0$,

$$
\dot{\vec{L}}=\sum_{i=1}^{N} \vec{r}_{i} \times\left(\alpha-\beta\left\|\vec{v}_{i}\right\|^{2}\right) \vec{v}_{i}-\sum_{i=1}^{N} \vec{r}_{i} \times k_{f} \vec{\nabla} U_{e s p}\left(\vec{r}_{i}\right)
$$

Asumiendo que el enjambre de partículas se encuentra cercano a un mínimo local, $\vec{\nabla}_{i} U_{\text {esp }}\left(\vec{r}_{i}\right) \approx 0$, entonces:

$$
\sum_{i=1}^{N} \vec{r}_{i} \times k_{f} \vec{\nabla}_{i} U_{e s p}\left(\vec{r}_{i}\right) \approx 0
$$

y reemplazando en (15),

$$
\dot{\vec{L}} \approx \sum_{i=1}^{N} \vec{r}_{i} \times\left(\alpha-\beta\left\|\vec{v}_{i}\right\|^{2}\right) \vec{v}_{i}
$$

De lo anterior se aprecia que si el enjambre de partículas se encuentra cerca a un mínimo local donde el gradiente del campo potencial es aproximadamente cero, entonces la formación presenta un movimiento circular alrededor del mínimo local con velocidad de cada partícula tendiendo a $\sqrt{\alpha / \beta}$.

\section{ANÁLISIS CUALITATIVO}

Sobre el comportamiento del enjambre se observa en primer lugar que el acoplamiento del sistema (enjambre), permite cambiar de un movimiento de traslación a rotación. En general, el enjambre exhibe un comportamiento dinámico de la siguiente manera:

- A partir de unas condiciones iniciales $(\vec{R} \neq 0)$, el enjambre va a invertir su energía en velocidades lineales, de manera que todos las partículas se mueven hacia la posición objetivo $O$.

- Una vez que llegan al objetivo, las partículas inician un ciclo limite alrededor de $O$.

Los pasos descritos anteriormente son ideales, por lo cual el verdadero enjambre exhibe un 
comportamiento más complejo que refleja los valores de los diferentes parámetros. En la Figura 1, se observa como la magnitud de las velocidades convergen al valor común de $\sqrt{\alpha / \beta}$. Los paneles de la Figura 2 muestran las diferentes etapas descritas anteriormente.

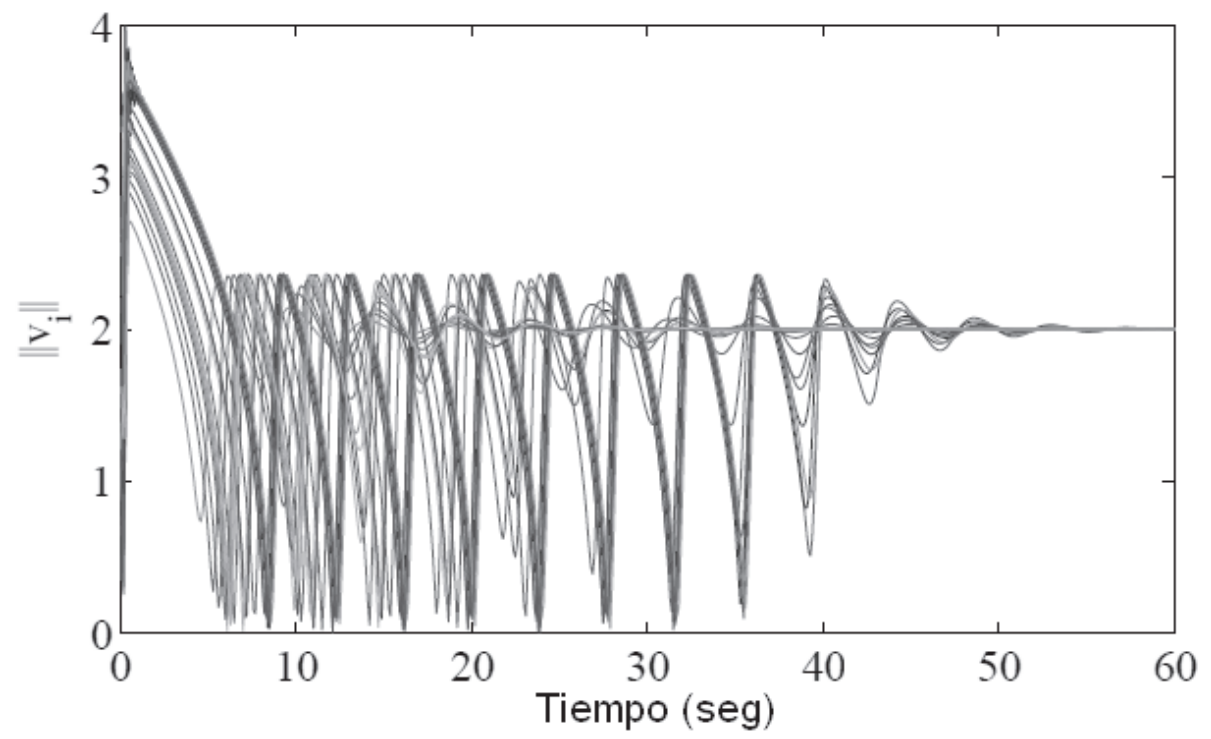

Figura 1. Evolución de la velocidad de cada partícula del enjambre. Se puede observar cómo las magnitudes de las velocidades convergen a un valor constante en este caso de $\sqrt{\alpha / \beta}=2$.

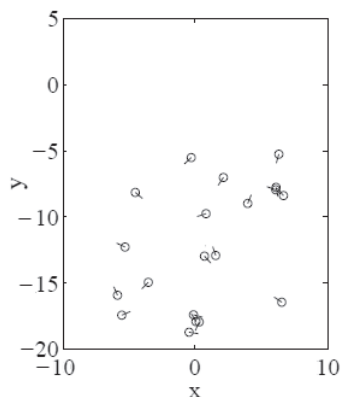

(a) Condición inicial.

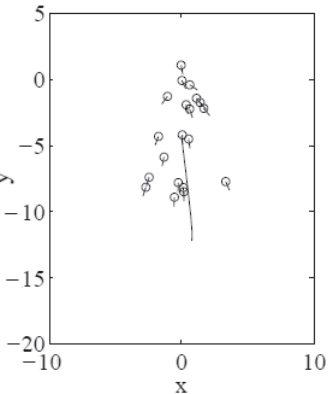

(b) Tiempo: $13 \mathrm{seg}$.

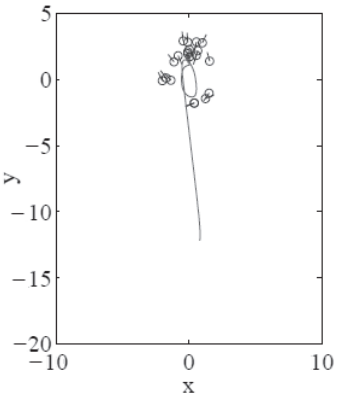

(c) Tiempo:15 seg.

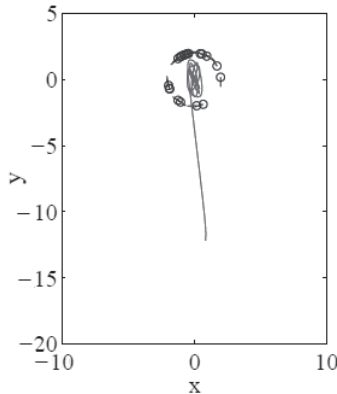

(d) Tiempo:60 seg.

Figura 2. Comportamiento típico del modelo de enjambre. La posición objetivo se establece en el origen. (a) Condición inicial. (b) Tiempo: $13 \mathrm{seg}$. El enjambre se pone en movimiento hacia el origen. (c) Tiempo 15 seg. El enjambre está cerca del origen. (d) Tiempo: 60 seg. El enjambre describe un círculo alrededor de su centro de masa.

\section{ALGORITMO PROPUESTO PARA EVADIR MÍNIMOS LOCALES}


El algoritmo desarrollado se denomina en inglés Vortex Swarm Path Planning Algorithm (VSPPA), lo cual se puede interpretar en español como Algoritmo de Planeación de Trayectorias con Partículas con Comportamiento de Vorticidad (APTPCV).

El algoritmo propuesto se basa en que el punto objetivo corresponde al mínimo global y adicionalmente, cuando el enjambre alcanza un mínimo local, presenta un movimiento circular alrededor de este punto. Teniendo en cuenta lo anterior, el algoritmo propuesto tiene por objeto aumentar la energía de propulsión del enjambre $\alpha$ cuando se alcanza un mínimo local y sólo se disminuye cuando el enjambre escapa de este punto. Para lograr lo anterior, se considera una variable $U_{\min }$ que se actualiza con el valor mínimo del campo potencial de $U_{\text {esp }}(\vec{R})$, lo cual se puede expresar como:

$$
U_{\text {min }}= \begin{cases}U_{e s p}(\vec{R}), & \mathrm{si} U_{\min } \geq U_{e s p}(\vec{R}) \\ U_{\text {min }}, & \mathrm{si} U_{\min }<U_{e s p}(\vec{R}) .\end{cases}
$$

La adición de la energía se logra con el factor de propulsión:

$$
\alpha=\alpha_{0}+\alpha_{e}
$$

Donde $\alpha_{0}$ es el factor de propulsión nominal y $\alpha_{e}$ representa una adición de energía. Este término debe ser positivo y acotado, lo cual implica:

$$
0 \leq \alpha_{e} \leq \alpha_{\max }
$$

En esta propuesta, la relación para $\alpha_{e}$ es:

$$
\frac{d \alpha_{e}}{d t}= \begin{cases}\tau_{c}, & \text { si } U_{\text {min }} \leq U_{e s p}(\vec{R}) ; \\ -\tau_{d}, & \text { si } U_{\text {min }}>U_{e s p}(\vec{R}) .\end{cases}
$$

Es de apreciar que al llegar el enjambre a un mínimo local $U_{\min } \leq U_{e s p}(\vec{R})$, el término $\alpha_{e}$ aumenta, incrementando así la energía de propulsión. Esta acción continuará hasta que las partículas escapen del mínimo local. El aumento de la energía viene dado por el parámetro $\tau_{c}$. Cuando el enjambre escapa del mínimo local, la energía adicional se libera con una tasa negativa $\tau_{d}$.

Para emplear el modelo dinámico del enjambre en el algoritmo de planeación de trayectorias, las ecuaciones diferenciales se pasan a tiempo discreto, considerando un intervalo de tiempo $\Delta t$, de tal forma que se tiene: 


$$
\begin{array}{r}
\vec{r}_{i}(n)=\vec{r}_{i}(n-1)+\vec{v}_{i} \Delta t \\
\vec{v}_{i}(n)=\vec{v}_{i}(n-1)+\left(\vec{F}_{p r o, i}+\vec{F}_{i n t, i}+\vec{F}_{e s p, i}+\vec{F}_{e s t, i}\right) \Delta t / m_{i}
\end{array}
$$

La contribución de $\alpha_{e}$ en tiempo discreto es:

$$
\alpha_{e}(n)=\alpha_{e}(n-1)+\Delta t \begin{cases}\tau_{c}, & \operatorname{si} U_{\text {min }} \leq U_{e s p}(\vec{R}) ; \\ -\tau_{d}, & \operatorname{si} U_{\text {min }}>U_{e s p}(\vec{R}) .\end{cases}
$$

Por último, el algoritmo propuesto para planear trayectorias, se puede apreciar a continuación:

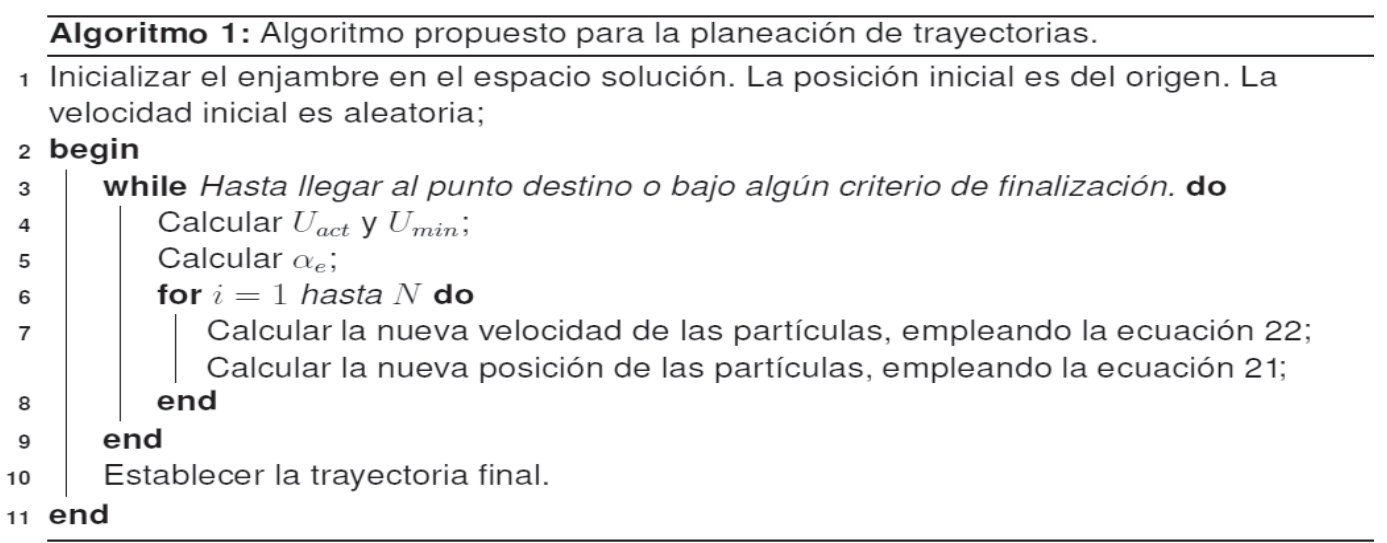

\section{CAMPOS POTENCIALES DE PRUEBA}

Para la prueba del algoritmo, se consideraron dos casos de importancia en la planeación de trayectorias de robots móviles. Uno se tiene cuando existe una barrera de obstáculos entre el punto inicial (PI), y el punto final (PF); a este tipo de configuración, se le denomina obstáculo tipo barrera [39]. Otro caso especial existe cuando el robot móvil debe pasar por un camino estrecho [1 1].

\subsection{CAMPO POTENCIAL CON OBSTÁCULO TIPO BARRERA}

La Figura 3 muestra el campo potencial con un obstáculo tipo barrera, que presenta un mínimo local. 
a) Campo potencial y trayectoria

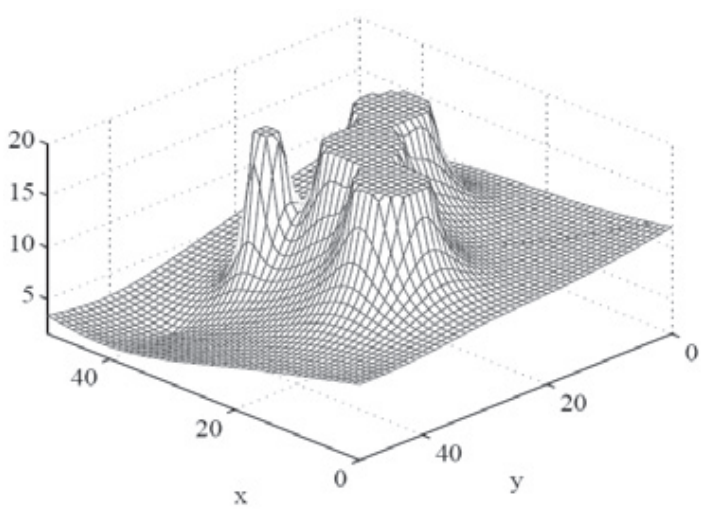

b) Contorno y trayectoria

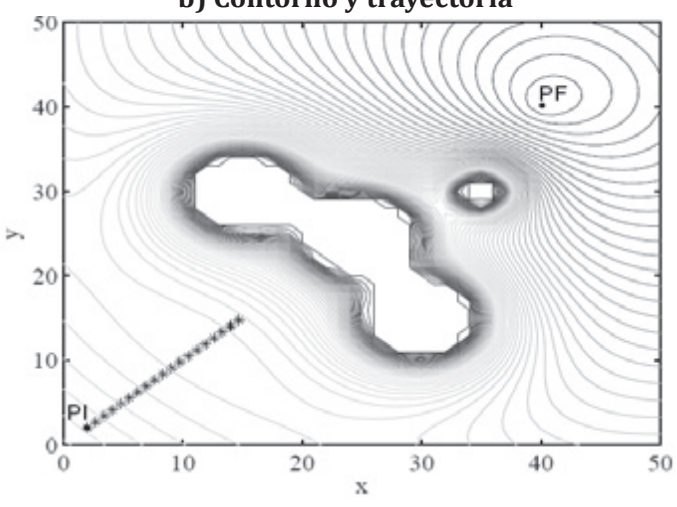

Figura 3. a) Campo potencial con la presencia de una barrera,

b) Contorno del campo potencial con la presencia de una barrera.

Tomando $D=1, N=20$ y $\beta=0.5$ en la Figura 4 se aprecian las trayectorias de las partículas y la posición promedio que corresponderá a la trayectoria final que seguirá el robot. Aunque se logra determinar una trayectoria desde el punto inicial al punto objetivo, esta puede ser no óptima, por lo cual, se puede considerar la necesidad de incorporar un algoritmo para suavizar las trayectorias irregulares y eliminar las trayectorias circulares.

a) Trayectorias

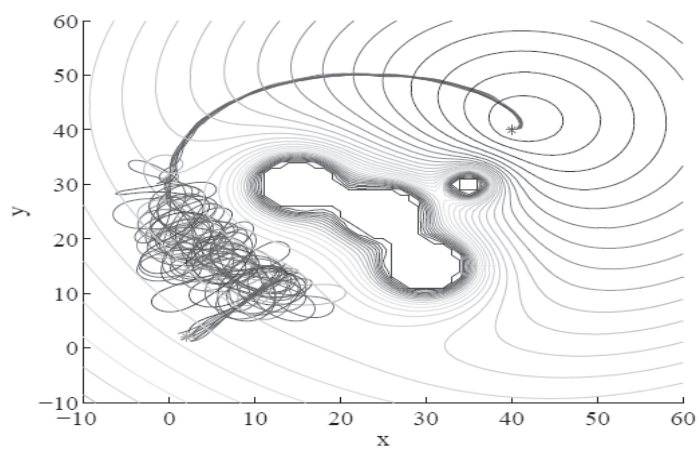

b) Trayectoria promedio

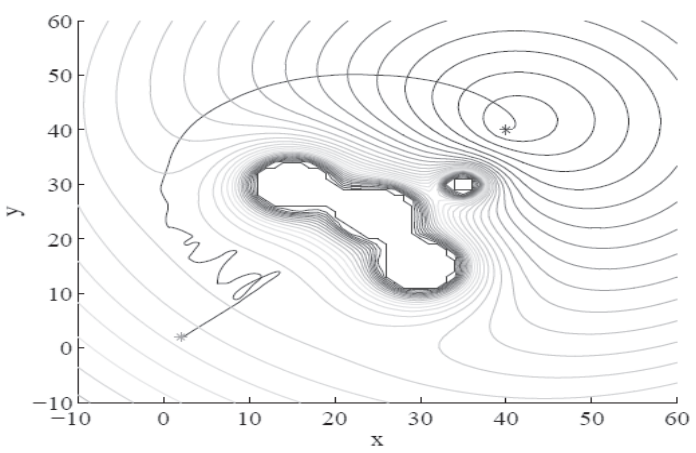

Figura 4. a) Trayectorias de las partículas,

b) Trayectoria promedio del enjambre, para $D=1, N=20$ y $\beta=0.5$

La Figura 5 muestra la energía que se adicionó para lograr que el enjambre escape del mínimo local. Es de apreciar que se llega al valor máximo de la energía de propulsión adicionada, y se mantiene en este valor hasta que el enjambre logra escapar del mínimo local. 


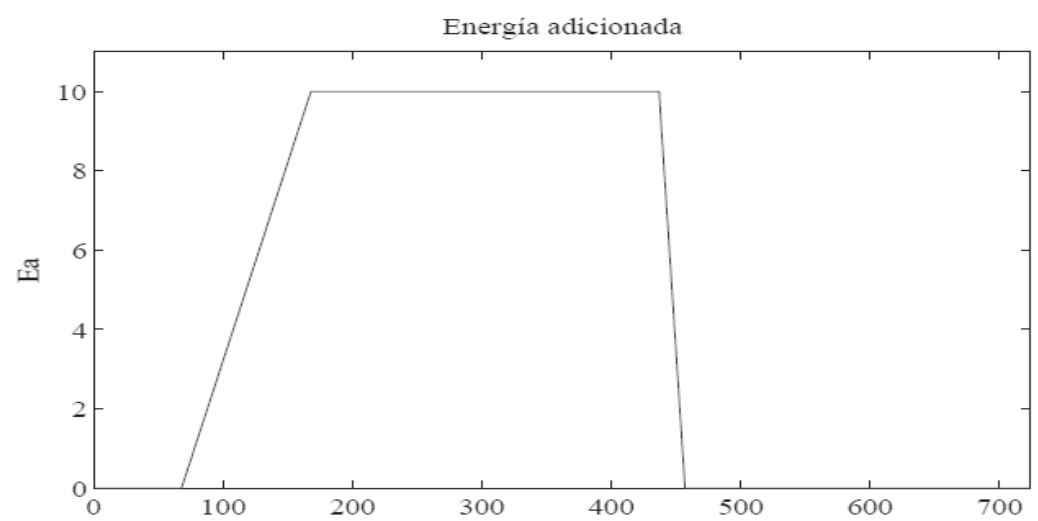

Figura 5. Energía adicionada para evadir el mínimo local, para $D=1, N=20$ y $\beta=0.5$

\subsection{CAMPO POTENCIAL CON PASANE ESTRECHO}

Cuando se tienen obstáculos muy próximos, se generan pasajes estrechos debido a las magnitudes de los campos potenciales asociados a los obstáculos. En este caso, cuando la magnitud de los campos potenciales es alta, se genera una barrera en el pasaje estrecho que dificulta la planeación de la trayectoria. Uno de los problemas que se presenta en esta configuración, es la oscilación de la trayectoria [1 11$]$.

En la Figura 6.a, se presenta el campo potencial generado con la presencia de un pasaje estrecho. El contorno de este campo potencial se puede apreciar en la Figura 6.b, donde se observa que en el inicio del pasaje estrecho se genera un mínimo local.

a) Campo potencial

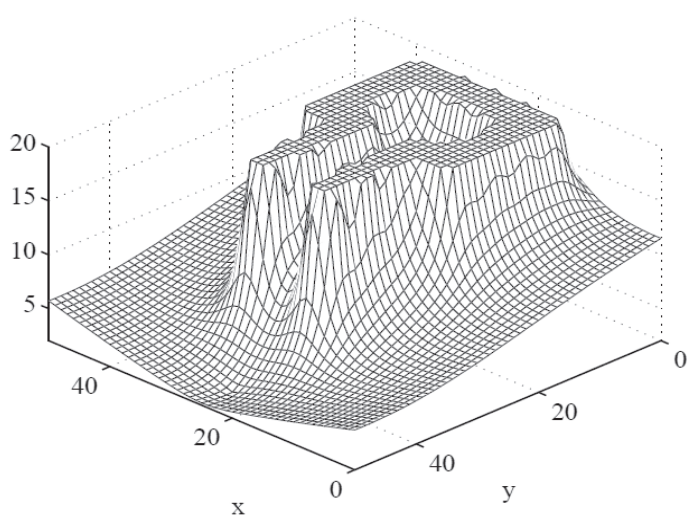

b) Contorno

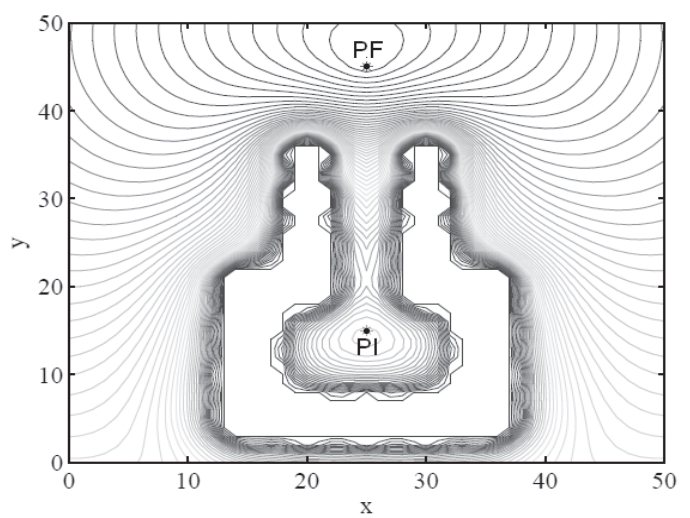

Figura 6. a) Campo potencial con la presencia de un pasaje estrecho, b) Contorno del campo potencial con la presencia de un pasaje estrecho. 
Considerando $D=1, N=20$ y $\beta=0.5$ en la Figura 7 se aprecia que el enjambre de partículas logra escapar del mínimo local y recorrer el pasaje estrecho, lo cual permite determinar una trayectoria desde el punto inicial al punto objetivo. Adicionalmente en la Figura 7, se observan oscilaciones en el pasaje estrecho, las cuales pueden hacer que el robot móvil colisione con los obstáculos.

a) Trayectorias

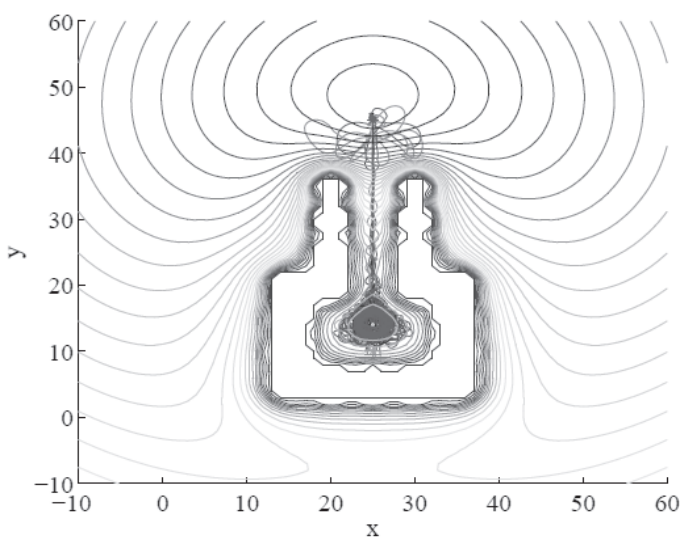

b) Trayectoria promedio

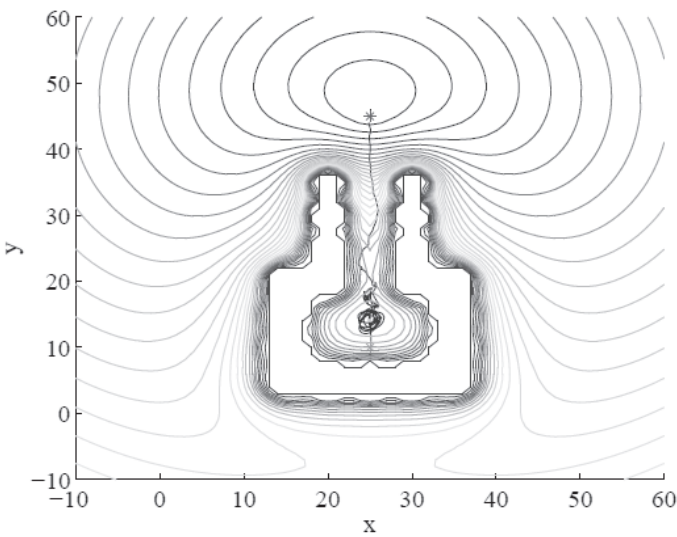

Figura 7. a) Trayectorias de las partículas, b) Trayectoria promedio del enjambre, para $D=1, N=20$ y $\beta=0.5$

En la Figura 8, se aprecia que la energía adicionada llega a su valor máximo y se mantiene en él, hasta que el enjambre logra salir del mínimo local. Lo anterior hace que la trayectoria generada presente movimientos circulares antes de escapar del mínimo local.

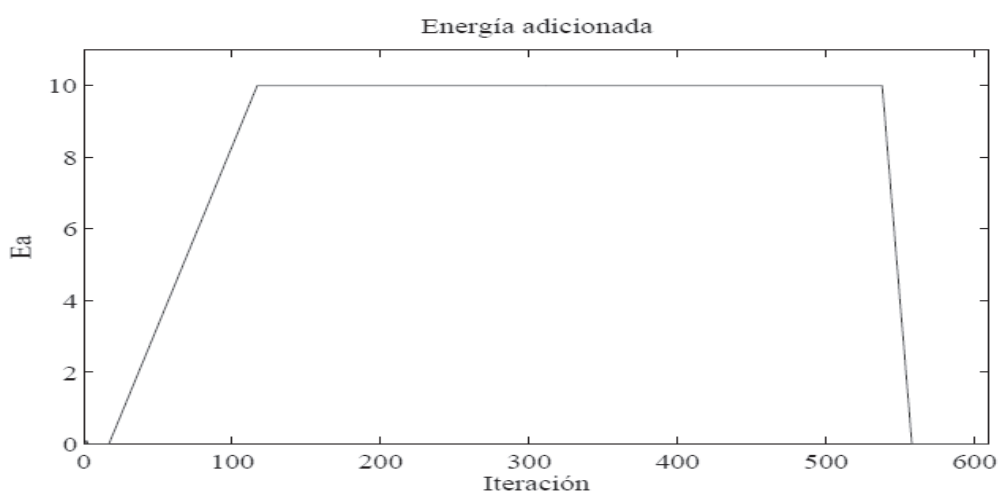

Figura 8: Energía adicionada para evadir el mínimo local, para $D=1, N=20$ y $\beta=0.5$ 


\section{ANÁLISIS DE RESULTADOS}

Con el fin de observar el comportamiento del algoritmo para diferentes parámetros, se consideraron los casos mostrados en la Tabla 1, donde se varia $D, N$ y $\beta$. El índice de comparación considerado consiste en el número de iteraciones requeridas para que el algoritmo determine la trayectoria desde el punto inicial hasta el final.

Tabla 1. Casos considerados para $D, N$ y $\beta$

\begin{tabular}{|l|c|c|c|c|c|c|c|c|}
\hline Caso & Caso 1 & Caso 2 & Caso 3 & Caso 4 & Caso 5 & Caso 6 & Caso 7 & Caso 8 \\
\hline $\mathrm{D}$ & 0 & 0 & 0 & 0 & 1 & 1 & 1 & 1 \\
\hline $\mathrm{N}$ & 10 & 10 & 20 & 20 & 10 & 10 & 20 & 20 \\
\hline$\beta$ & 0.2 & 0.5 & 0.2 & 0.5 & 0.2 & 0.5 & 0.2 & 0.5 \\
\hline
\end{tabular}

Para determinar si existe diferencia significativa en los resultados, se realiza un análisis estadístico, utilizando la metodología descrita en la Figura 9 [44, 45]. Esta metodología permite elegir entre pruebas paramétricas y no paramétricas en función, si se cumplen los supuestos de normalidad y homocedasticidad (igualdad de varianza).

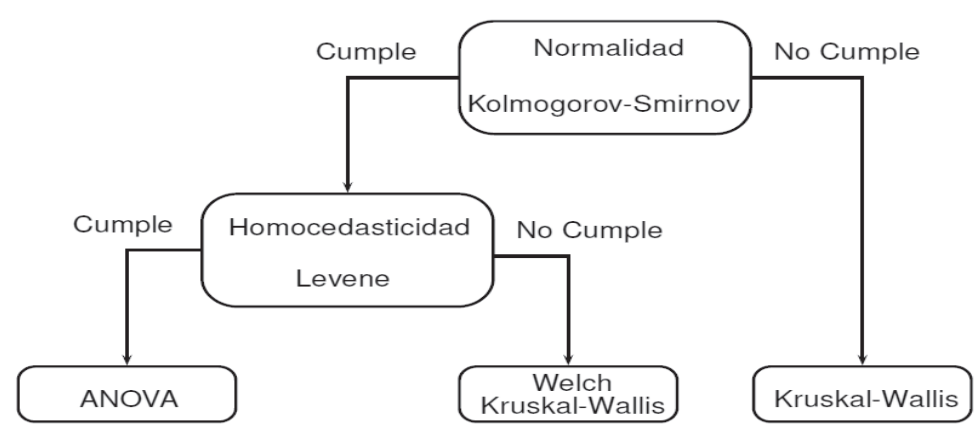

Figura 9. Metodología para establecer la prueba de hipótesis para realizar.

\subsection{CAMPO POTENCIAL CON OBSTÁCULO TIPO BARRERA}

Para el campo potencial con un obstáculo tipo barrera, los resultados para 50 ejecuciones del algoritmo con las diferentes configuraciones de parámetros, se pueden apreciar en la Tabla 2.

Tabla 2. Resultados para el campo potencial con obstáculo tipo barrera

\begin{tabular}{|l|r|r|r|r|r|r|r|r|}
\hline Caso & \multicolumn{1}{|c|}{ Caso 1 } & \multicolumn{1}{c|}{ Caso 2 } & Caso 3 & Caso 4 & \multicolumn{1}{c|}{ Caso 5 } & \multicolumn{1}{c|}{ Caso 6 } & \multicolumn{1}{c|}{ Caso 7 } & \multicolumn{1}{c|}{ Caso 8 } \\
\hline Media & 481.18 & 461.12 & 536.50 & 442.10 & 536.36 & 803.66 & 542.00 & 997.38 \\
\hline VAR & 17769.3 & 11811.7 & 27216.4 & 1036.3 & 53165.7 & 138183.3 & 48291.1 & 498435.3 \\
\hline STD & 133.30 & 108.68 & 164.97 & 32.19 & 230.58 & 371.73 & 219.75 & 706.00 \\
\hline MR & 163.80 & 130.09 & 207.90 & 129.24 & 175.55 & 298.51 & 181.67 & 317.24 \\
\hline
\end{tabular}


Al realizar la prueba de normalidad para los datos recolectados, se obtiene un $p$-value de $1.4996 \times 10^{-20}$; por lo tanto, para determinar si existe variabilidad significativa entre los diferentes casos, se realiza la prueba de Kruskal-Wallis y se obtiene un $p$-value de 0 que indica que existen diferencias entre los casos considerados. Para determinar los grupos que presentan diferencias, se hace la prueba no paramétrica de Bonferroni y se obtienen los resultados mostrados en la Figura 10.

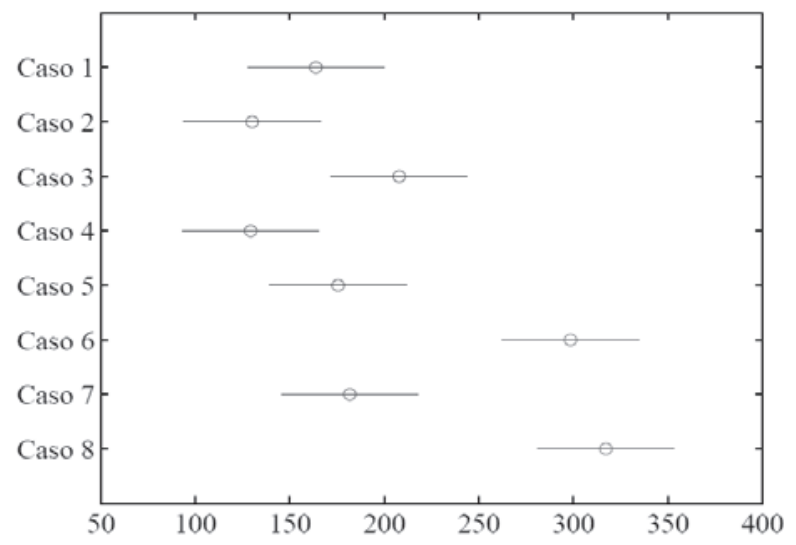

Figura 10. Resultados de la comparación para los casos con variación de parámetros.

El listado de los casos que presentan diferencias significativas son:

- Caso 1: con 6 y 8

- Caso 2: con 3, 6 y 8

- Caso 3: con 2, 4, 6 y 8

- Caso 4: con 3, 6 y 8

- Caso 5: con 6 y 8

- Caso 6: con 1, 2, 3, 4, 5 y 7

- Caso 7: con 6 y 8

- Caso 8: con 1, 2, 3, 4, 5 y 7

Los resultados anteriores muestran que la diferencia más significativa se presenta en los casos 6 y 8 , en relación con los otros casos. Para este campo potencial, no se puede establecer una relación directa entre el comportamiento del algoritmo y los parámetros considerados.

\subsection{CAMPO POTENCIAL CON PASANE ESTRECHO}

Para este campo potencial los resultados de 50 ejecuciones del algoritmo con las diferentes configuraciones de parámetros se pueden apreciar en la tabla 3. 
Tabla 3. Resultados para el campo potencial con pasaje estrecho.

\begin{tabular}{|l|c|c|c|c|c|c|c|c|}
\hline Caso & Caso 1 & Caso 2 & Caso 3 & Caso 4 & Caso 5 & Caso 6 & Caso 7 & Caso 8 \\
\hline Media & 279.98 & 942.70 & 295.38 & 911.16 & 264.68 & 1145.26 & 268.40 & 890.84 \\
\hline VAR & 1733.2 & 299316.8 & 2463.7 & 341174.7 & 1484.5 & 299908.1 & 734.4 & 104195.2 \\
\hline STD & 41.63 & 547.10 & 49.64 & 584.10 & 38.53 & 547.64 & 27.10 & 322.79 \\
\hline MR & 112.57 & 290.69 & 128.48 & 283.26 & 80.40 & 319.83 & 91.54 & 297.23 \\
\hline
\end{tabular}

Al realizar la prueba de normalidad de Kolmogorov-Smirnov, se tiene un $p$-value de $3.9839 \times 10^{-19}$ que rechaza de esta forma, la hipótesis nula de normalidad de los datos; por lo tanto, se emplea la prueba de Kruskal-Wallis para determinar si existe diferencia significativa entre los datos tomados con los diferentes parámetros; al realizar esta prueba, se tiene un $p$-value de 0 , por lo cual se procede a realizar la comparación entre grupos, empleando la prueba de Bonferroni, y se obtienen los resultados mostrados en la Figura 11.

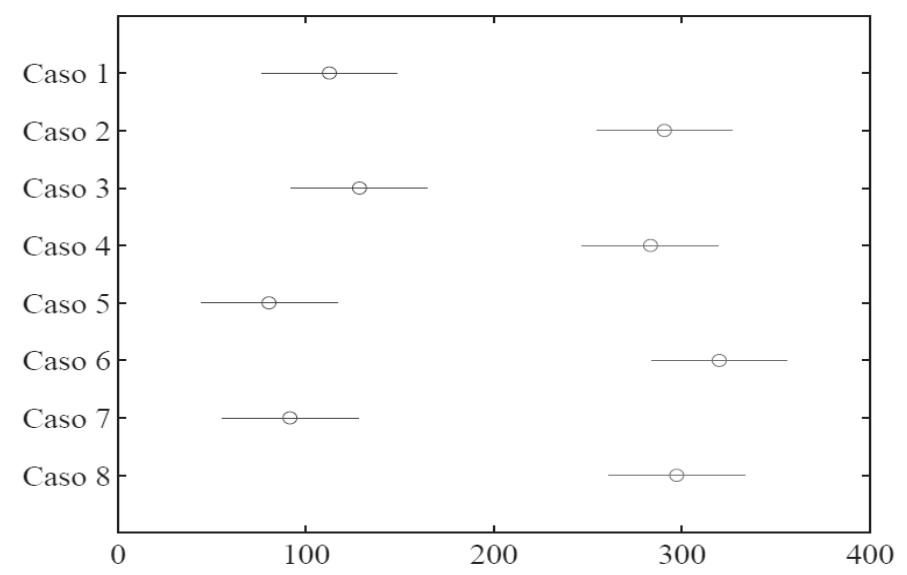

Figura 11 Resultados de la comparación para los casos con variación de parámetros

Con estos resultados, se observa que los casos 1, 3, 5 y 7 presentan diferencias significativas con los casos 2, 4, 6 y 8. Lo anterior muestra que para este grupo de experimentos, el parámetro que presenta mayor influencia en el desempeño del algoritmo es $\beta$.

\section{CONCLUSIONES}

La selección del modelo se realizó, buscando tener una expresión compacta con pocos términos y que permitiera describir comportamientos de enjambre y desplazamientos uniformes y movimientos circulares, los cuales se aprecian en diferentes grupos de seres vivos. 
Para observar las características del modelo, se hizo un análisis sin considerar el componente estocástico; con este análisis, se muestra el comportamiento del movimiento circular de las partículas bajo ciertas características de los parámetros del modelo.

Con los resultados cualitativos y cuantitativos, se apreció que para los campos potenciales considerados, el algoritmo propuesto logra evadir mínimos locales y permite establecer una trayectoria entre el punto origen y destino.

Aunque en los casos considerados se logra determinar una trayectoria desde el punto inicial al punto objetivo en algunas situaciones se observa la presencia de trayectorias con irregularidades y movimientos circulares, lo cual puede producir trayectorias no óptimas. Considerando lo anterior, en un trabajo futuro se puede desarrollar un algoritmo para suavizar las trayectorias irregulares y eliminar las trayectorias circulares.

Con el análisis estadístico de los datos, se validan las observaciones efectuadas para los diferentes experimentos. Los parámetros que se modificaron para observar el comportamiento del algoritmo fueron seleccionados, considerando los análisis reportados del modelo y las simulaciones previas del mismo.

En los resultados se apreció que el parámetro asociado a la fuerza disipativa, presenta un efecto considerable en el algoritmo en comparación con el número de partículas y la magnitud del ruido. Esta característica puede utilizarse para mejorar el desempeño del algoritmo.

En un trabajo futuro, se puede proponer una estrategia de control para el factor de propulsión con la cual se pueda evadir mínimos locales en forma más eficiente. Una alternativa adicional para mejorar el algoritmo, consiste en modificar de forma dinámica el factor asociado con el campo potencial, de tal forma que el enjambre pueda escapar rápidamente de mínimos locales.

En trabajos futuros, se podría considerar otros modelos de partículas autopropulsadas y partículas brownianas, para implementar el algoritmo propuesto. También se puede aplicar el concepto presentado en este documento, para el desarrollo de algoritmos de optimización.

\section{REFERENCIAS BIBLIOGRAFÍCAS}

[1] Udo Erdmann, Werner Ebeling, Schimansky-Geier Lutz, Ordemann Anke, and Moss Frank, (2008). Active brownian particle and random walk theories of the motions of zooplankton: application to experiments with swarms of daphnia. In: Journal of Theoretical Biology 9.

[2] Hsin Ya-Chieh, (2006). Emergence of vortex swarming in daphnia. Term Paper for Emergent State of Matter, Spring.

HELBERT EDUARDO ESPITIA CUCHANGO, JORGE IVÁN SOFRONY ESMERAL 
[3] Werner Ebeling, (2002). Nonequilibrium statistical mechanics of swarms of driven particles. Elsevier: Physica A.

[4] Levine Herbert, Rappel Wouter-Jan, and Cohen Inon, (2000). Self-organization in systems of self-propelled particles. In: Physical Review E 63.

[5] D’Orsogna M.R., Chuang Y.L., Bertozzi A.L., and Chayes L.S., (2006). Self-propelled particles with soft-core interactions: patterns, stability, and collapse. In: Physical Review Letters, PRL 96.

[6] Werner Ebeling, and Udo Erdmann, (2002). Nonequilibrium Statistical Mechanics of Swarms of Driven Particles. In: Physica A: Statistical Mechanics and its Applications, Vol. 314, Issues 1-4, pp. 92-96.

[7] Udo Erdmann, Werner Ebeling, and Alexander S. Mikhailov, (2005). Noise-Induced Transition from Translational to Rotational Motion of Swarms. In: Physical Review E.

[8] Abdel Wahid M.H.M., and Mclnnes Colin R., (2008). Wall following to escape local minima for swarms of agents using internal states and emergent behavior. International Conference of Computational Intelligence and Intelligent Systems ICCIIS.

[9] Gómez-Bravo F., Cuesta F., y Ollero Baturone Aníbal, (2003). Planificación de trayectorias en robots móviles basada en técnicas de control de sistemas no holónomos. XXIV Jornadas de Automática.

[10] Ollero Baturone Aníbal, (2001). Robótica Manipuladores y robots móviles. Marcombo.

[1 1] De Los Santos De La Rosa Erik Adolfo, (2004). Heurística para la generación de configuraciones en pasajes estrechos aplicada al problema de los clavos. Tesis de Maestría en Ciencias con Especialidad en Ingeniería en Sistemas Computacionales, Universidad de las Américas Puebla.

[12] Khaled Belghith, Kabanza Froduald, Hartman Leo, and Nkambou Roger, (2006). Anytime Dynamic Path-Planning with Flexible Probabilistic Roadmaps Khaled Belghith. Proceedings IEEE International Conference on Robotics and Automation.

[13] Bennewitz Maren, Burgard Wolfram, and Thrun Sebastian, (2002). Finding and Optimizing Solvable Priority Schemes for Decoupled Path Planning Techniques for Teams of Mobile Robots. In: Robotics and Autonomous Systems.

[14] Poty A., Melchior P., and Oustaloup A., (2004). Dynamic Path Planning For Mobile Robots Using Fractional Potential Field. First International Symposium on Control, Communications and Signal Processing. 
[15] Tu Jianping, and Yangt Simon, (2003). Genetic Algorithm Based Path Planning for a Mobile Robot. Proceedings of the IEEE International Conference on Robotics \& Automation.

[16] Nilsson J. Nils, (1969). A Mobile Automaton: An Application of Artificial Intelligence Techiques. Proc. of the 1st. International Joint Conference on Artificial Intelligence.

[17] Segovia Armando, and Rombaut Michkle, (1993). Path Finding from a Spot Image for a Mobile Robot. Intelligent Vehicles 93 Symposium.

[18] Brooks Rodney A., (1983). Solving the Find-Path Problem by Good Representation of Free Space. IEEE Transactions on Sysyems Man and Cybernetics.

[19] Lozano Peréz Tomás, (1983). Spatial planning: a configuration space approach. IEEE Transactions on computers.

[20] Sleumer Nora H., and Tschichold Gürman Nadine, (1999). Exact Cell Decomposition of Arrangements used for Path Planning in Robotics. Swiss Federal Institute of Technology.

[21] Barraquand Jérome, Langlois Bruno, and Latombe Jean-Claude, (1992). Numerical Potential Field Techniques for Robot Path Planning. IEEE Transactions on Systems, Man and Cybernetics.

[22] Kavraki Lydia E., and Latombe Jean-Claude, (1998). Probablistic Roadmaps for Robot. In Practical Motion Planning in Robotics: Current Approaches and Future Directions, John Wiley.

[23] Overmars Mark H., and Svestka Petr, (1994). A probabilistic learning approach to motion planning. Proceding in Workshop on Algorithmic Foundations of Robotics.

[24] Lindemann Stephen R., and La Valle Steven M., (2004). Steps Toward Derandomizing RRTs. In: IEEE Fourth International Workshop on Robot Motion and Control.

[25] Fujimori Atsushi, Nikiforuk Peter, and Gupta Madan, (1997). Adaptive Navigation of Mobile Robots with Obstacle Avoidance. IEEE Transactions on Robotics and Automation.

[26] Gemeinder M., and Gerke M., (2003). GA-based Path Planning for Mobile Robot Systems Employing an Active Search Algorithm. Applied Soft Computing.

[27] Liu Guan-Yu, and Wu Chia-Ju, (2001). A Discrete Method for Time-Optimal Motion Planning of a Class of Mobile Robots. In: Journal of Intelligent and Robotic Systems.

[28] Xiao Jing, Michalewicz Zbigniew, Zhang Lixin, and Trojanowski Krzysztof, (1997). Adaptive Evolutionary Planner/Navigator for Robots. IEEE Transactions on Evolutionary Computation. 
[29] Porta García Miguel Ángel, (2007). Planeación de trayectorias para robótica móvil mediante optimización por colonia de hormigas. Tesis de Maestría en Ciencias en Sistemas Digitales, Instituto Politécnico Nacional, Tijuana, México.

[30] Coelho Leandro dos Santos, and Sierakowski Cezar Augusto, (2006). Bacteria Colony Approaches With Variable Velocity Applied to Path Optimization of Mobile Robots. ABCM Symposium Series in Mechatronics, Vol. 2.

[31] Sierakowski Cezar A., and Coelho Leandro dos Santos, (2005). Study of two swarm intelligence techniques for path planning of mobile robots. 16th IFAC World Congress, July.

[32] Khatib O., (1986). Real-time obstacle avoidance for manipulators and mobile robots. In: International Journal of Robotic Research, Vol. 5 (1), 90p.

[33] Krogh B., (1984). A generalized potential field approach to obstacle avoidance control. ASME Conference of Robotic Research: The Next Five Years and Beyond. Bethlehem, Pennsylvania.

[34] Khosla P., and Volpe R., (1988). Superquadric artificial potentials for obstacle avoidance and approach. IEEE Internaitional Conference on Robotics and Automation., Philadelphia, April 26-28.

[35] Kim J.O., and Khosla P.K., (1991). Real-time obstacle avoidance using harmonic potential functions. IEEE Conference on Robotics and Automation, Sacramento, pp.790-796.

[36] Ge S.S., and Cui Y.J., (2000). New potential functions for mobile robot path planning. IEEE Transactions on Robotics and Automation, Vol. 16 (5), 615p.

[37] Connolly C.I., and Burns J.B., (1994). Path Planning using Laplace's equation. IEEE Conference on Robotics and Automation.

[38] Rimon E., and Koditchek D.E., (1992). Exact robot navigation using artificial potential functions. IEEE Transactions on Robotics and Automation, Vol. 8 (5).

[39] Lee Leng-Feng, (2004). Decentralized motion planning within an artificial potential framework (APF) for cooperative payload transport by multi-robot collectives. Master Of Science Thesis, University of New York at Buffalo, December.

[40] Prahlad Vadakkepat, Kay Chen Tan, and Wang Ming-Liang, (2000). Evolutionary Artificial Potential Fields and Their Application in Real Time Robot Path. Proceedings of the Congress on Evolutionary Computation. 
[41] Xi-Yong Zou, and Jing Zhu, (2003). Virtual local target method for avoiding local minimum in potential field based robot navigation. In: Journal of Zhejiang University - Science A.

[42] Chengqing Liu, Ang Marcelo, Krishnan Hariharan, and Yong Lim Ser, (2000). Virtual Obstacle Concept for Local-minimum-recovery in Potential-field Based Navigation. Proceedings of the IEEE International Conference on Robotics \& Automation.

[43] Mikhailov Alexander S., and Zanette Damián H., (1999). Noise-induced breakdown of coherent collective motion in swarms. In: Physical Review E 60:44, pp.4571-4575.

[44] Arriaza Gómez A.J., Fernández Palacín F.M., López Sánchez A., Muñoz Márquez M., Pérez Plaza S., y Sánchez Navas A., (2008). Estadística básica con R y R-Commander. Servicio de Publicaciones de la Universidad de Cádiz.

[45] Cagnina Leticia Cecilia, (2010). Optimización mono y multiobjetivo a través de una heurística de inteligencia colectiva. Tesis de Doctorado en Ciencias de la Computación, Universidad Nacional de San Luis, Argentina. 